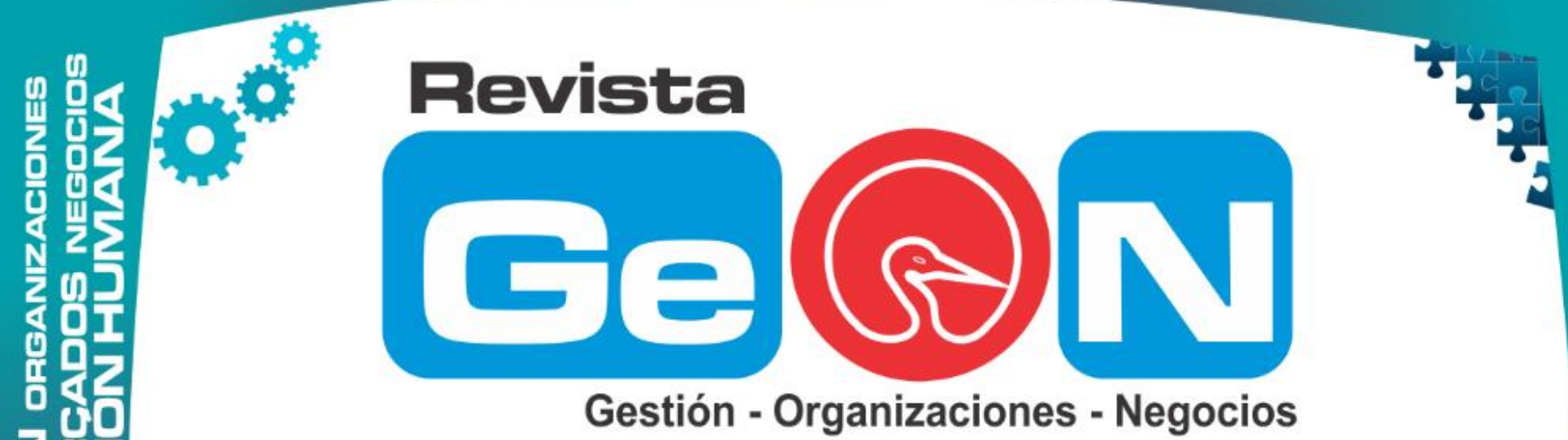

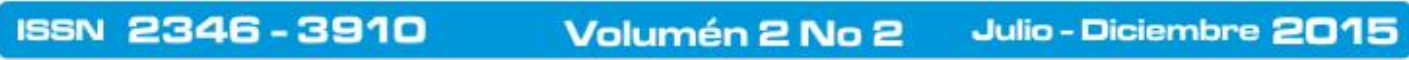

Revista Electrónica de la Facultad de Ciencias Económicas de la Universidad de los Llanos

1. 3 II

440

VILLAVICENCIO - COLOMBIA

$>2$

64

2

2 平品

70

ব)

展

o

ii III

0 U

ใด

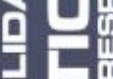

居的

임

Iiin in

照

只

造

iil 2

if in

4 吕

2

400

付 iI
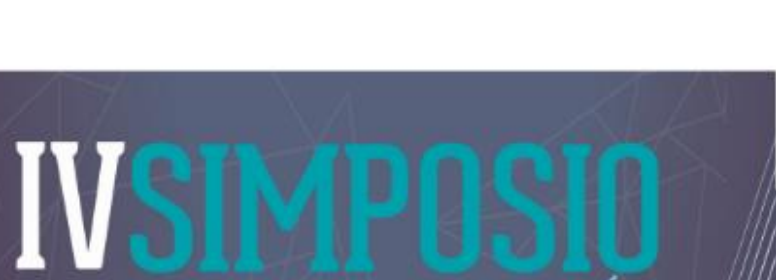

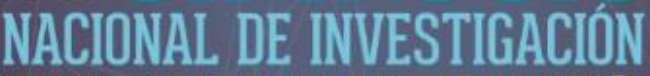

EN MARKETING
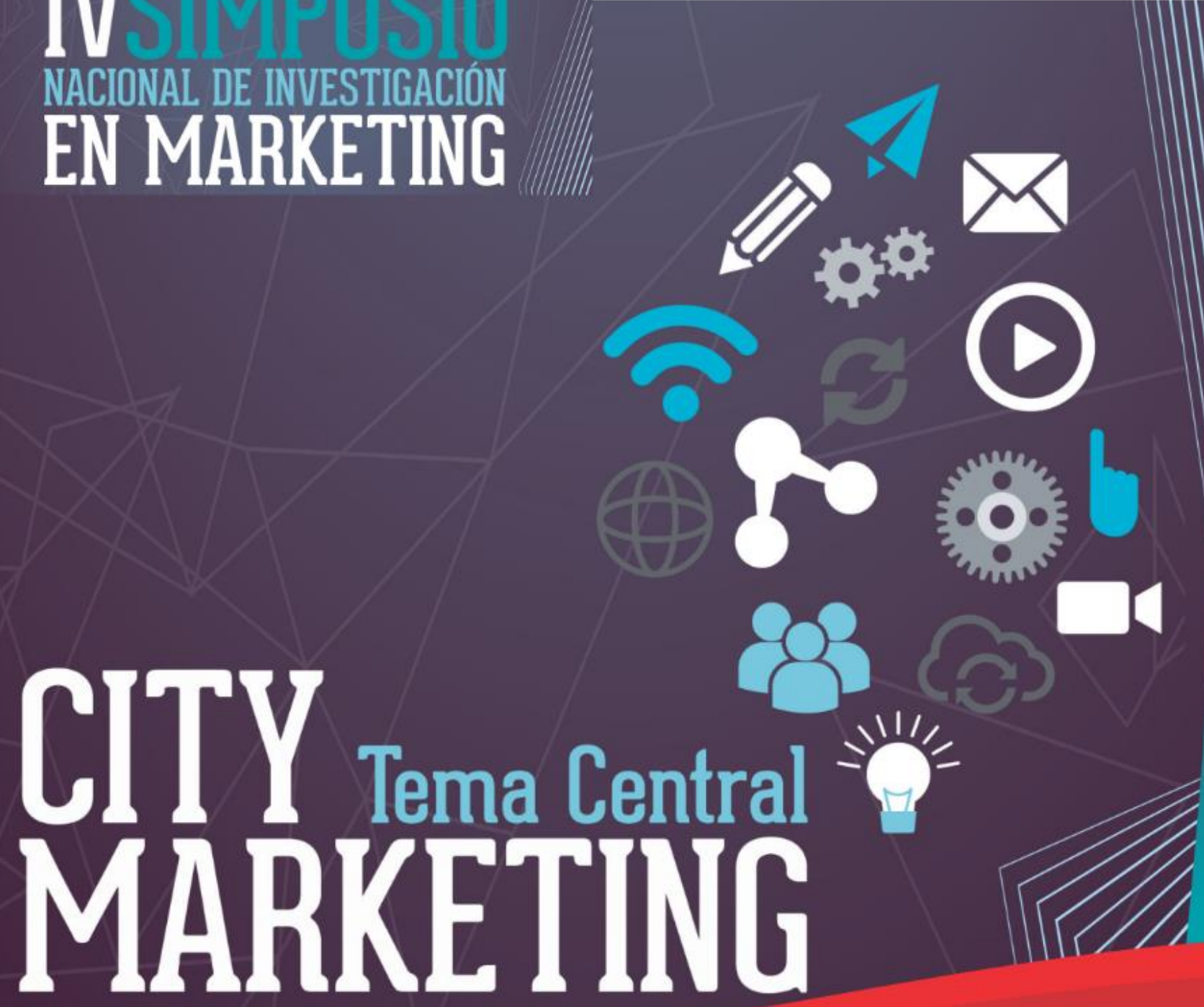

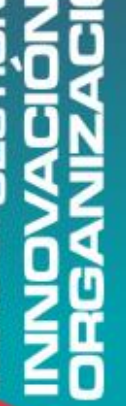
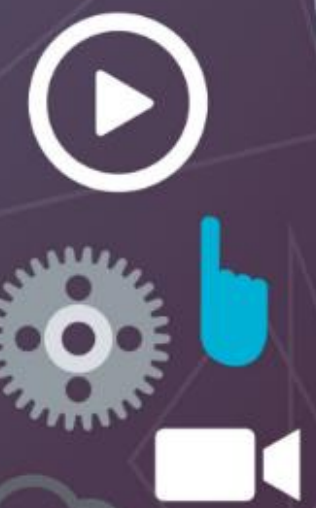


\section{Un algoritmo evolutivo para la solución de problemas en Marketing - Henry Lamos - Luis Alferez}

\author{
Henry Lamos D \\ $\mathrm{PhD}$ Matematicas Aplicadas \\ hlamos@unab.edu.co \\ Luis Gerardo Alferez \\ Magister en Administración \\ lalferez@unab.edu.co \\ @ lgalferez
}

\section{Resumen}

En el trabajo se presentan tres problemas del área del Marketing que se modelan por medio de la optimización combinatoria. Los problemas de optimización combinatoria pertenecen a la clase de problemas que se consideran NP-Hard. Se presenta un enfoque de solución mediante la meta-heurística evolutiva EPSO “Optimización por enjambre de partículas evolutivas". El TDPs consiste en determinar una división de un conjunto de unidades ubicadas en un territorio que cumple con los criterios múltiples como la compacidad, la conectividad y el equilibrio en términos de clientes y la demanda del producto; el siguiente problema es selección y diseño de un conjunto de productos para una línea de productos (PLD); el problema consiste en determinar los niveles de los atributos par producto que maximice la elección; y el último problema que se estudia es el VRPSPD resuelve el problema de distribución de la cadena de abarrotes y alimentos, determina la flota de vehículos que minimiza el tiempo de recorrido. Los problemas juegan un importante papel en la gestión del área del marketing que no debe ser ignorado en sus decisiones.

\section{Palabras clave}

EPSO; Particle Swarm Optimization Evolution; TDP; Diseño Territorio; Enrutamiento vehicular; Diseño de Productos; NP-hard; Clientes; Demanda.

\begin{abstract}
This work of investigation is to develop a solution to three problems in the work area of marketing that are modeled by combinatorial optimization. Through the development of a framework and building a toolbox in Matlab, implementing the meta-heuristic EPSO "Evolutionary Swarm Optimization particles." The TDPs is to determine a division of a set of units located in a territory that meets multiple criteria such as compactness, connectivity and balance in terms of customer and product demand. The PLDP consist in determine the specific attribute levels for each multiattribute product in a set of products to maximize the resulting product line's share of choice, and the VRPSPD resolve problem the determine the vehicle fleet for minimize the sum of travel time with the restriction the vehicle must have enough capacity for transporting the commodities to the delivered and those ones picked-up customers and returning depot. All problems constituting an important support that should not be ignored by those responsible for the activities related to the commercial area as it becomes a tactical decision for the company.
\end{abstract}

\section{Keywords}

EPSO; Particle Swarm Optimization Evolution; TDP; Territory Design; Vehicle routing; conjoint analysis; NP-hard; Customers; Demand.

\section{Introducción}

El Diseño de Territorios permite a las empresas dedicadas a las ventas y servicios de bienes y productos mejorar la competitividad con el objetivo de mejorar la rentabilidad. En la actualidad las empresas implementan estrategias innovadoras para mejorar su nivel de servicio al cliente. Una actividad importante a considerar es la distribución de territorios comerciales, la cual permite a la organización ajustarse 
adecuadamente a las necesidades de los clientes, mejorando la cobertura y ayudando a la compañía a mantener su fuerza de ventas, tiempos de viaje y costos bajo control.

El Problema de Diseños de Territorios (TDP por sus siglas en inglés) consiste en determinar una agrupación de manzanas, o clientes individuales que se denominan territorios $o$ unidades básicas (UBs), en un área geográfica determinada, en un número fijo de territorios de manera que se cumplan una serie de requerimientos de planificación impuestos por la empresa. Un estudio general sobre problemas de diseño de territorios se puede hallar en Kalcsics et al. (2005). El problema ha sido resuelto mediante técnicas de optimización exactas y aproximadas, para modelos recientes $\mathrm{y}$ algoritmos se puede consultar los trabajos de Aerts et al. (2003) and Xiao (2006).

El Análisis Conjunto es un método que permite estimar la estructura de preferencias de los consumidores, dadas las evaluaciones globales hacia un conjunto de alternativas caracterizadas en términos de los niveles de los diferentes atributos del producto o servicio, Green y Rao (2001).

Por medio del análisis conjunto de acuerdo a [Hair y otros, 1995] se puede:

1. Determinar la contribución de las variables explicativas (niveles) y sus respectivos valores (utilidades) a la determinación de las preferencias del consumidor,

2. Construir un modelo válido de valoración del consumidor útil en la predicción del nivel de aceptación por parte del consumidor de cualquiera de las combinaciones de los atributos, incluso aquellos que no hayan sido evaluados originalmente por los consumidores.

Entre los problemas importantes de los Gerentes de Marketing que manejan múltiples productos se encuentra el problema de diseño de un conjunto de productos para una línea de productos. Una gran variedad de aplicaciones se encuentra en la literatura, como por ejemplo, en seguros de salud, en planes de telefonía celular, servicio de pizzas, automóviles, etc. Uno de los autores pioneros en la investigación de diseño de una línea de productos a partir de los datos partworth obtenidos mediante un análisis conjunto fue Zufryden (1977, 1982), quien formulo el problema como un problema de programación entera. Diferentes funciones objetivos se han propuestos, entre las que se encuentran, la maximización de beneficios, la utilidad ponderada, y la cuota de elección.

El término "óptimo" se refiere a determinar una línea de productos que maximice las cuotas de mercado de elección de la línea (SOC, por sus siglas en inglés) en una muestra representativa de los consumidores. El objetivo es determinar los niveles de atributos específicos para cada producto de la línea para maximizar el número de encuestados cuya utilidad de al menos un nuevo producto excede la utilidad de reserva del entrevistado.

El Problema Ruteo de Vehículos (VRP por sus siglas en inglés) consiste en la construcción de rutas desde un depósito hasta un número determinado de clientes ubicados en un espacio geográfico, con el fin de ofrecer el servicio de entrega de bienes con una flota de vehículos. El diseño de sistemas de distribución efectivos puede representar un ahorro de costos significativo a las empresas. Un problema que se estudia en el Marketing es el VRPSPD, una de las aplicaciones del VRPSPD se encuentra en los sistemas de distribución de las cadenas de abarrotes y alimentos, donde cada tienda de abarrotes usualmente presenta demandas tanto de entrega (comida o bebidas frescas) como de recolección (alimentos vencidos o botellas vacías) y es atendida en una sola visita del proveedor. Con esto, los minoristas pueden negociar la devolución del exceso de productos no vendidos a los productores con efectos beneficiosos para ambas partes.

Los tres problemas presentados en el este trabajo son problemas de optimización combinatoria de tipo NP-hard. Encontrar soluciones exactas requiere de un tiempo de 
cómputo significativamente grande debido a la complejidad algorítmica de tipo exponencial del problema, Cordeau et. al (2002). Si bien los problemas pueden ser abordados mediante métodos exactos para instancias pequeñas Amico et al. (2006), los casos reales de aplicación de éstos son siempre problemas de gran tamaño. Por tal motivo, se han aplicado técnicas heurísticas y metaheurísticas que proporcionan buenas soluciones al problema de optimización en tiempos de cómputo razonables.

En este trabajo se propone un algoritmo para la solución de los problemas discutidos (TDPs, PLD, VRPSPD) utilizando un enfoque de optimización de enjambre de partículas (PSO). Los autores han obtenidos resultados para el TDP, VRPSPD mediante el algoritmo PSO y en esta en vía de construcción para el PLD. Resultados del algoritmo propuesto se puede hallar en Lamos et al $(2013,2014)$.

El presente trabajo está organizado de la siguiente manera: En la sección 2 se presentan la formulación matemática de los tres problemas; en la sección 3 se define el método PSO y su implementación para determinar la solución; en la sección 4, se presentan algunos resultados computacionales y finalmente, las conclusiones son presentadas en la sección 5

\section{Definición de los problemas}

El TDP se puede formular mediante el grafo $G=(V, E)$, donde, $\mathrm{V}$ es el conjunto de UBs(Unidad Básica de Control)- y $E$ es el conjunto de $\operatorname{arcos}$ que representan adyacencia entre manzanas. Para cada nodo $j$ en el conjunto $V$ se definen las coordenadas geográficas $\left(x_{1}{ }^{j}, x_{2}{ }^{j}\right)$, y dos atributos o actividades: número de clientes y volumen de ventas. La distancia Euclidiana, $d_{i j}$ se calcula entre cada par de UBs $i$ y $j$. El problema consiste en determinar una partición de territorios $\mathfrak{F}$ de unidades básicas de control, de forma que cada nodo pertenezca sólo a un territorio (asignación exclusiva).

Adicionalmente, la compañía busca territorios balanceados con respecto al número de clientes y demanda de producto. Se define el tamaño de un territorio $V_{k} \subset V$ con respecto a la actividad $a$, como la suma de los valores de actividad asociados a las unidades básicas que conforman el territorio, esto es $w^{(a)}=$ $\sum_{i \in V_{k}}\left(w_{i}^{(a)}\right)$, aquí $k$ es el índice del territorio, $a \in A=\{1,2\}, \mathrm{y}_{i}{ }^{(a)}$ es el valor asociado a la actividad a en el nodo $i \in V$. Debido a la estructura discreta del problema y a la restricción de asignación exclusiva, es prácticamente imposible tener territorios perfectamente balanceados, es decir, territorios con exactamente el mismo tamaño con respecto a cada actividad. Entonces, con el fin de modelar el balance, se introduce un parámetro de tolerancia $\tau^{a}$. Este parámetro mide la desviación relativa con respecto al tamaño deseado en la actividad $a, a \in \mathrm{A}$. El valor deseado representa el tamaño promedio de los territorios y está dado por $\mu^{a}=w^{a}(V) / p$. Otra restricción importante es la de conexidad, es decir, por cada $i$ y $j$ asignadas al mismo territorio debe existir una ruta entre ellos y ésta debe estar totalmente contenida en el territorio. Además, con el fin de lograr compacidad, las UBs del mismo territorio deben estar tan cerca una de la otra como sea posible. La medida que se estudia se basa en el objetivo del problema de p-centro, Salazar et al (2011).

A continuación se presenta el modelo de programación entera. Se define el conjunto $N^{i}$, para todo $i$ en $V$ como el conjunto de todas las UBs adyacentes a la UB $i$, es decir,

$$
N^{i}=\{j \in V:(i, j) \in E \vee(j, i) \in E\}
$$

Las variables de decisión se definen como:

$=\left\{\begin{array}{c}1 \text { si la UB } i \text { es asignada al territorio con centro en } j \\ 0 \text { en otro caso }\end{array}\right.$

Note que $x_{i i}=1$ implica que la UB $i$ es un centro territorial. El modelo matemático MTDP (TDP basado en $p$-mediana) se define a continuación.

(MTDP) 
$=\sum_{j \in V} \sum_{i \in V}^{\operatorname{minimizar} z} d_{i j} x_{i j}$

$$
\begin{aligned}
& \text { sujeta a } \sum_{i \in V} x_{i i} \\
& =p
\end{aligned}
$$$$
\sum_{i \in V} x_{i j}=1 \quad j
$$$$
\in V
$$

$\sum_{j \in V} w_{j}^{a} x_{i j} \geq\left(1-\tau^{a}\right) \mu^{a} x_{i i} \quad i \in V, a$

$$
\in A
$$

$\sum_{j \in V} w_{j}^{a} x_{i j} \leq\left(1-\tau^{a}\right) \mu^{a} x_{i i} \quad i \in V, a$

$$
\in A
$$

$$
\sum_{j \in \cup_{v \in S} \frac{N^{v}}{S}} x_{i j}-\sum_{j \in S} x_{i j} \geq 1-|S|
$$

$i \in V, S$

$$
\subset \frac{V}{N^{i} \cup\{i\}}
$$

$$
x_{i j} \in\{0,1\} \quad i, j
$$$$
\in V
$$

El objetivo (1) representa una medida de dispersión basada en el objetivo del $p$ MP. En este sentido, minimizar dispersión es equivalente a maximizar compacidad. La restricción (2) garantiza la creación de exactamente $p$ territorios. Las restricciones (3) representan la asignación exclusiva de las UBs.

Las restricciones (4)-(5) representan el balance con respecto a cada actividad y establecen que el tamaño de cada territorio debe estar dentro de un rango de variación (determinado por $\tau^{a}$ ) con respecto al tamaño promedio. La conexidad de los territorios está dada por las restricciones (6). Estas últimas son similares a las restricciones de eliminación de subrutas (subtours) en el problema del agente viajero.

La definición del problema PLDP consiste en que dado el número de ítems en la línea del producto $\mathrm{M}$, cada uno con $\mathrm{K}$ atributos $\mathrm{y}$ sus respectivos niveles $L_{k}$ (el número de niveles del atributo $\mathrm{k}=1,2, . . \quad \mathrm{K})$. El número total de encuestado es $\mathrm{S}$. Los parámetros $u_{k j}{ }^{s}$ representan las utilidades parciales (partworts), para $\mathrm{j}=1,2, \ldots L_{k}$, en el atributo $\mathrm{k}$ y respondiente s (Wang et al. 2006).

Sean las variables de decisión $x_{j k m}, y_{s m}$ y $y_{s}$ de la siguiente forma: $x_{j k m}=1$ si el nivel $j$ del atributo $k$ es elegido para el producto $m$ y 0 en caso contrario. La variable $y_{s m}=1$ si el producto $m$ le da al consumidor $s$ una utilidad más alta que su utilidad reserva y 0 en caso contrario, por último $y_{s}=1$ si la utilidad de reserva del consumidor $s$ es excedida por al menos un producto en la línea y 0 en caso contrario. Las ecuaciones que aparecen en (8)(11) es la formulación de programación entera para el problema de la línea de producto SOC.

$$
\max z=\sum y_{m}
$$

$$
\begin{gathered}
\text { sujeta a } \sum_{j} x_{k j m}=1 \quad k=1,2, \ldots K ; m \\
=1,2, \ldots M(9)
\end{gathered}
$$

$$
\begin{gathered}
\sum_{m=1}^{M} y_{s m} \geq y_{s}, \quad \mathrm{~m}=1, . . \mathrm{M} \\
\sum_{k=1}^{K} \sum_{j=1}^{L_{k}} u_{k j}{ }^{s} x_{k j m} \geq h_{s} y_{s m}, s=1,2, \ldots S, m \\
=1,2, \ldots M
\end{gathered}
$$

El PLDP consiste en maximizar el número de individuos que tienen utilidad para al menos un producto en la línea de productos que supera su utilidad de reserva. El conjunto de 
restricciones modelan elementos que se deben cumplir en el mundo real, esto es, la restricción (9) asegura que sólo un nivel de cada atributo se selecciona para cada producto; la restricción (10) permite asegurar que si se cumple que $y_{s}=0$ cuando ningún producto constituido tiene una utilidad superior a utilidad de reserva del entrevistado.

La restricción (11) se asegura que $y_{s m}=1$ sólo si el producto $\mathrm{m}$ proporciona una utilidad más alta que la utilidad de reserva para $s$ cliente. La línea de problema de diseño de productos SOC se ha determinado que es NP-Hard (Kohli et al, 1989).

Por último, el VRPSPD se puede formular de la siguiente manera (Ai et al, 2009):

Sea $\boldsymbol{G}=(\boldsymbol{V}, \boldsymbol{A})$ un grafo en donde $V=$ $\left\{v_{0}, v_{1}, \ldots, v_{n}\right\}$ es un conjunto de nodos, y $A=$ $\left\{\left(v_{i}, v_{j}\right) \mid v_{i}, v_{j} \in V, i \neq j\right\}$ es un conjunto de arcos que conectan al nodo $i$ con el nodo $j$. Asociada al conjunto $A$ se encuentra una matriz de distancias $\left(d_{i j}\right)$ y una matriz de tiempo de viaje $\left(t_{i j}\right)$. El nodo $v_{\mathrm{o}}$ representa un depósito en el cual se encuentra un número de $m$ vehículos homogéneos, mientras que los demás nodos corresponden a $n$ clientes por atender. Cada cliente tiene cantidades no negativas de recogida $\boldsymbol{p}_{\boldsymbol{i}}$, de entrega $\boldsymbol{q}_{\boldsymbol{i}}$ y un tiempo de servicio $\boldsymbol{S}_{\boldsymbol{i}}$. Cada vehículo tiene un costo fijo $f$, un costo variable por unidad de distancia $g$, capacidad $Q$, y duración límite de servicio $D$. El problema VRPSPD consiste en el diseño de un grupo de máximo $m$ rutas de tal manera que:

(1) Cada ruta empieza y termina en el depósito;

(2) cada cliente es visitado una vez por un solo vehículo;

(3) la carga total del vehículo en el trayecto entre dos clientes (considerando la demanda de entrega $\boldsymbol{q}_{\boldsymbol{i}}$ y de recogida $\boldsymbol{p}_{\boldsymbol{i}}$ ) no excede la capacidad de carga total del vehículo $(Q)$;
(4) la duración total de cada ruta (incluyendo el tiempo de viaje y de servicio) no excede el límite previamente establecido $D ; \mathrm{y}$

(5) el costo total de ruteo es mínimo.

El VRPSPD también se puede formular como problema de programación lineal entera mixta.

\section{Variables de decisión}

$X_{i j k}$ es una variable binaria que indica si el arco $(i, j)$ es recorrido o no por el vehículo $k$

$X_{i j k}=1$ si el vehículo $k$ recorre el arco $(i, j)$

$X_{i j k}=0$ si el vehículo $k$ no recorre el arco $(i, j)$

$Y_{i j k}$ Carga del vehículo $k$ que recorre el arco $(i, j)$

$\delta_{i k}$ Tiempo de inicio de servicio del cliente $i$ efectuado por el vehículo $k$

\section{Función Objetivo}

Minimizar:

$Z=f \sum_{k=1}^{m} \sum_{j=1}^{n} X_{0 j k}+$

$g \sum_{i=0}^{n} \sum_{j=1}^{n+1} \sum_{k=1}^{m} d_{i j} * X_{i j k}$

Sujeto a:

$\sum_{k=1}^{m} X_{i j k}=1 \quad$ para $1 \leq j \leq n$

$\sum_{j=1}^{n+1}=X_{i j k}$ para $1 \leq i \leq n, 1 \leq k \leq m$

$\sum_{j=1}^{n} X_{0 j k} \leq 1$ para $1 \leq k \leq m$

$\delta_{i k}+S_{i}+t_{i j}+\delta_{j k} \leq\left(1-X_{i j k}\right) M \quad$ para $0 \leq$ $i \leq n, 1 \leq j \leq n, 1 \leq k \leq m$ (16)

$\delta_{n+1, k}-\delta_{0 k} \leq D$ para $1 \leq k \leq m$ 
$y_{i j k} \leq x_{i j k} * Q \quad$ para $0 \leq i \leq n, 1 \leq j \leq n+1$, $1 \leq k \leq m$

$\sum_{j=1}^{n} y_{0 j k}=\sum_{j=1}^{n} q_{j} * \sum_{i=0}^{n} x_{i j k} \quad$ para $1 \leq k \leq$ m (19)

$\sum_{i=0}^{n} y_{i j k}+\left(p_{j}-q_{j}\right) * \sum_{i=0}^{n} x_{i j k}=\sum_{i=1}^{n+1} y_{i j k}$

para $1 \leq j \leq n, 1 \leq k \leq m$ (20)

$x_{i j k} \in\{0,1\}$, para $0 \leq i \leq n, 1 \leq j \leq n+1$,

$1 \leq k \leq m$

$y_{i j k} \geq 0, \quad$ para $\quad 0 \leq i \leq n, 1 \leq j \leq n+1$,

$1 \leq k \leq m$

$\delta_{i j k} \geq 0, \quad$ para $\quad 0 \leq i \leq n, 1 \leq j \leq n+1$,

$1 \leq k \leq m$

En la formulación anterior, la función objetivo (12) representa el costo total de transporte teniendo en cuenta los costos fijos y variables asociados a los vehículos. La restricción (13) indica que cada cliente es visitado únicamente por un vehículo; la restricción (14) indica que cada vehículo que llega a un nodo (cliente) debe salir de él. Esta restricción debe además garantizar que cada vehículo retorne al depósito luego de haber visitado el último cliente de la ruta, por lo cual debe considerarse un arco desde el nodo $n$ hasta el nodo $n+1$, siendo este último el depósito al final del recorrido. La restricción (15) indica que un vehículo puede servir como máximo a una ruta. La restricción (16) relaciona el tiempo de inicio de servicio entre un cliente y otro. La restricción (17) indica la relación entre el primer y último cliente en cada ruta, es decir, establece el límite de duración del servicio.

La restricción (18) indica la máxima capacidad $(Q)$ del vehículo $k$ mientras sirve al cliente $j$ después de haber servido al cliente $i$. Por otro lado, la restricción (19) asegura que todas las entregas a clientes partan desde el depósito, y la restricción (20) se encarga de equilibrar la carga del vehículo $k$ después del servicio a un cliente.

Finalmente, las restricciones (21)-(23) indican el dominio de las variables de decisión.

\section{Optimización de enjambre de partículas}

En esta sección, se presenta el algoritmo de optimización de enjambre de partículas para resolver los problemas (TDP, PLDP y VRPSPD) descritos previamente; la representación de la solución y el método de decodificación de la solución (Ai et al, 2009)

\section{Algoritmo PSO}

El PSO es un algoritmo poblacional de búsqueda basado en la simulación del comportamiento social de aves de una bandada (Kennedy et al, 1995). Formalmente, un enjambre puede ser definido como un grupo de individuos que se comunican directa o indirectamente entre ellos, actuando en su respectivo ambiente. La interacción entre individuos resulta en estrategias colectivas de resolución de problemas.

En el PSO los individuos se conocen como partículas que pertenecen a un espacio de búsqueda $\boldsymbol{X} \subseteq \boldsymbol{R}^{\boldsymbol{n}}$. Los cambios de posición de las partículas dentro del espacio de búsqueda están basados en las tendencias sicosociales de individuos de emular el éxito de otros individuos, es decir, influenciados por la experiencia o conocimiento de sus vecinos. Como resultado, el comportamiento colectivo que surge es que todo el enjambre converge al estado que es mejor para todos sus miembros.

El algoritmo original está constituido por un enjambre de $l$ partículas, con masa y volumen despreciables, que se mueve sobre un espacio de $H$ dimensiones. La habilidad de una partícula para encontrar soluciones se representa por su vector velocidad que dirige el movimiento de la partícula de una posición a otra. Para realizar este movimiento, el enjambre cuenta con términos asociados a la 'memoria' que tiene cada partícula acerca de su experiencia y la de sus vecinos, de manera que la partícula actualiza su velocidad con respecto al comportamiento del enjambre. El modelo PSO combina dos tipos de aprendizaje para cada partícula del enjambre y un objetivo fijo que es común a cada una de ellas. En el PSO básico el primer tipo de 
aprendizaje de la partícula está asociado a la experiencia personal que ésta desarrolla en la medida en que se desplaza por el espacio de búsqueda conocido como pbest y es el vector denotado en (24).

$\Psi_{l}=\left[\psi_{l 1}, \psi_{l 2}, \ldots, \psi_{l H}\right]$

Este comportamiento recibe el nombre de "comportamiento cognitivo", y se define como la posición que arroja el mejor valor de la función objetivo entre todas las posiciones que han sido visitadas por la partícula.

El segundo tipo de aprendizaje corresponde al término que se relaciona con el aprendizaje que obtiene la partícula de su interacción con el enjambre, conocido como Gbest, y es el vector denotado en (25).

$\Psi_{g}=\left[\psi_{g 1}, \psi_{g 2}, \ldots, \psi_{g H}\right]$

Éste es denominado "comportamiento social" que se relaciona con la experiencia del vecindario de cada partícula, y se define como la posición que arroja el mejor valor de la función objetivo entre las posiciones que han sido visitadas por todas las partículas.

La posición de una partícula $l$ en la iteración $t$ es el vector que se denota en (26), donde $l=$ $1, \ldots, L$ y $t=1, \ldots, T$.

$\Theta_{l(\tau)}=\left[\theta_{l 1}, \theta_{l 2}, \ldots, \theta_{l H}\right]$

La actualización del vector de posición está asociada a la velocidad, la cual se compone de los términos de aprendizaje de la partícula y el peso inercial de ésta.

La velocidad para cada partícula $l$ del enjambre en la iteración $t$ es el vector denotado en (27):

$\Omega_{l}(\tau)=\left[\omega_{l 1}, \omega_{l 1}, \ldots, \omega_{l H}\right]$

La posición de la partícula $l$, cambia en cada iteración $t$ de acuerdo a (28):
$\Theta_{l}(\tau+1)=\Theta_{l}(\tau)+\Omega_{l}(\tau+1)$

Para utilizar el algoritmo aplicado a problemas de optimización, se requieren métodos de codificación que permitan reemplazar el 'objetivo' del enjambre por la función objetivo del problema. A partir del cambio en el desempeño de esta función, el método permite que la velocidad direccione el movimiento de todo el enjambre. En este direccionamiento se refleja el conocimiento de todas las partículas del enjambre y el efecto de la inercia de cada una de ellas.

El algoritmo aplicado para resolver por ejemplo el VRPSPD se presenta a continuación. En primer lugar se inicializa el enjambre y se evalúa el desempeño de la función fitness mediante el método de codificación. De esta manera se actualiza la información de los comportamientos del enjambre. Posteriormente se actualiza la velocidad y la posición, se evalúa el criterio de parada y continúa con la siguiente iteración.

$u \quad$ Número aleatorio distribución uniforme $[0,1]$

$\mathrm{w}(\mathrm{t})$ Peso inercial de la t-ésima iteración

$c_{p} \quad$ Constante de aceleración de la posición pbest

$\mathrm{c}_{\mathrm{g}}$ Constante de aceleración de la posición gbest

$\theta_{\max }$ Valor de posición máximo

$\theta_{\min } \quad$ Valor de posición mínimo

$\mathrm{R}_{\mathrm{l}} \quad$ El l-ésimo conjunto de rutas de vehículos

$\mathrm{Z}\left(\Theta_{\mathrm{l}}\right)$ Valor de desempeño de $\Theta_{\mathrm{l}}$

y del método de Optimización de Enjambre de Partículas (PSO) para solucionarlo. Se aplica una representación de la solución y un método de decodificación para implementar el PSO al VRPSPD. El método de decodificación inicia transformando una partícula en una lista de prioridades de clientes para entrar a las rutas y 
una matriz de prioridades de vehículos para servir cada cliente.

Las rutas de los vehículos son construidas con base en la lista de prioridad de clientes y en la matriz de prioridad de vehículos. El algoritmo es evaluado usando 18 instancias disponibles en la literatura. Los resultados computacionales muestran que el método propuesto arroja buenas soluciones para problemas de 100, 200 y 400 clientes, sin embargo, no mejora los resultados obtenidos por otros métodos metaheurísticos para resolver el VRPSPD

\section{Análisis de los resultados computacionales}

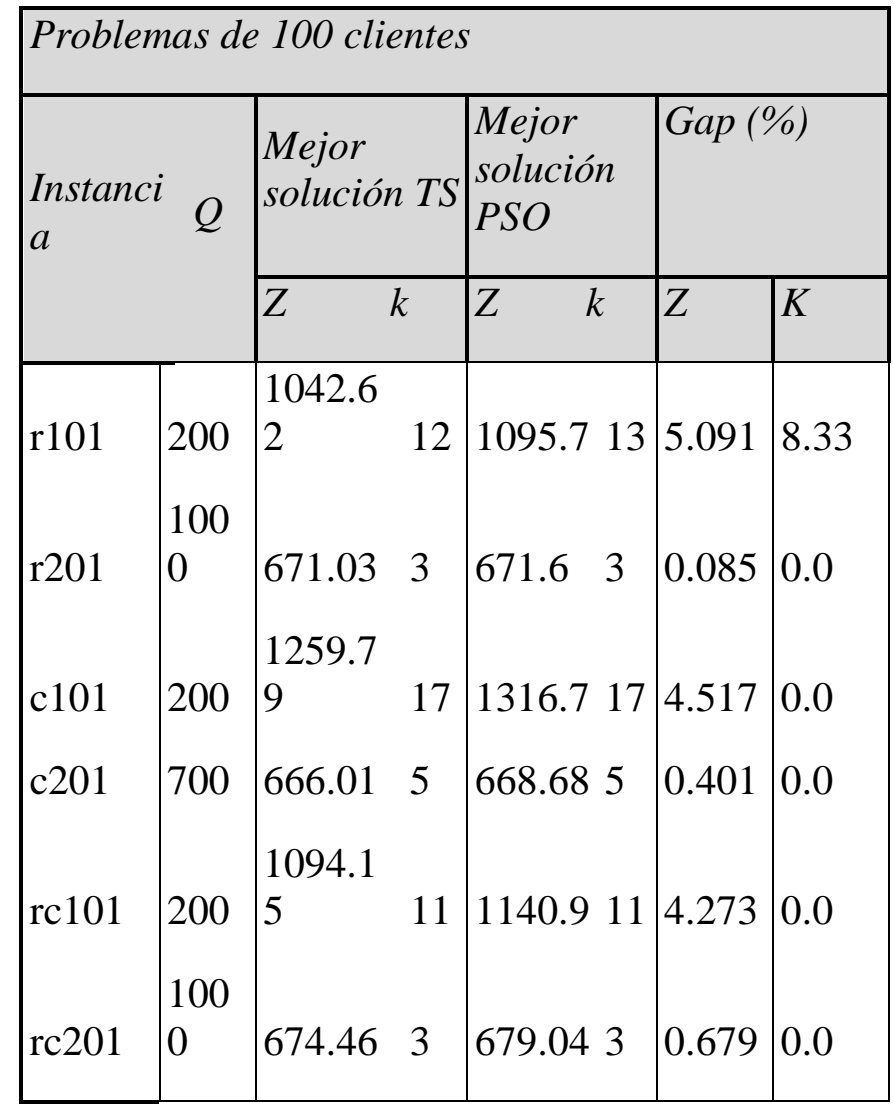

Tabla 1 Resultados para instancias de 100 clientes

Los experimentos numéricos efectuados para evaluar el desempeño del PSO se llevaron a cabo aplicando el método en un conjunto de instancias. Se presenta algunos resultados solo para el VRPSPD. El algoritmo se implementó en MatLab, versión R2012a, en un equipo con procesador Intel Core i5 con 4 GB de memoria RAM instalada.
Las soluciones obtenidas fueron comparadas con el método Búsqueda Tabú (TS) propuesto por Tang et al.

En los resultados obtenidos para las instancias de 100 clientes, se pudo observar que la desviación entre los resultados obtenidos y los resultados obtenidos por el método TS, no superan el $6 \%$ para la función de costos.

El algoritmo EPSO para resolver el problema de Diseño Territorial Comercial fue programado en Matlab versión R2012a y ejecutado en un equipo con procesador Intel Core i3 y $4 \mathrm{~GB}$ de RAM instalada (la solución se encuentra en Lamos et al (2014).

\section{Conclusiones}

En el trabajo se presentan tres problemas importantes en el mundo del Marketing, el problema de diseño de territorios con el objetivo de rediseñar territorios de venta en forma periódica que impacta de manera positiva en la planeación estratégica y operativa de cualquier empresa dedicada a la venta y distribución de productos.

Las ciudades de Colombia tienen una gran cantidad de tiendas, las cuales presentan demandas de entrega y recogida de bienes, lo que hace que se deba tener un sistema de distribución de las cadenas de abarrotes y alimentos que sea óptimo. Además, dado que las leyes fuerzan a las empresas a tomar responsabilidad por la vida útil de sus productos, estas se ven obligadas a colectar productos usados con el fin de darles un apropiado procesamiento o disposición final.

Para el problema de ruteo de vehículos se desarrolló un toolbox de matbal que permite hallar las soluciones, sin embargo, es necesario implementarlos en un lenguaje de programación para ser usado en otros ambientes diferentes al que se desarrolló; respecto al problema de diseño de territorios, se validó mediante instancias de tamaño pequeño, en estos momentos hay un estudiante de maestría para validaron a una escala de instancias de mayor tamaño. El 
desarrollo del software para el problema de diseño de una línea de productos se encuentra en una etapa inicial.

Es de señalar que la consecución de data del mundo real en nuestra región es de gran dificultad, por lo tanto, su validación mediante datos reales es bastante compleja.

La importancia de llevar el software desarrollado al mundo real es una tarea en que los autores se encuentran comprometidos con el fin de brindar una herramienta de apoyo a la gestión de las pequeñas empresas.

\section{Referencias}

Aerts JCJH, Eisinger E, Heuvelink GBM, Stewart TJ (2003). "Using linear integer programming for multi-site land use allocation". Goegr Anal 35(2): 148-169.

Ai T. J, V. Kachitvichyanukul (2009). “A particle swarm optimization for the vehicle routing problem with simultaneous pickup and delivery", Comput. Oper. Res., vol. 36, no. 5, pp. 1693-1702.

Amico M. D., Righini. and Salani M (2005). “ A branch-and-price approach to the vehicle routing problem with simultaneous distribution and collection".

Cordeau J.F, Gendreau M, Laporte G, Potvin J.-Y, Semet F (2002). "A guide to vehicle routing heuristics", J. Oper. Res. Soc., vol. 53, no. 5, pp. 512-522.

Green P. E., A. M. Krieger, Y. J. Wind. (2001). "Thirty years of conjoint analysis: Reflections and prospect"s. Interfaces 31(3) 56-73

Kalcsics J, Nickel S, Schoreder M (2005). "Towards a unified territorial design approach: applications, algoritms, and GIS integration". Top 13(1): 1-56

Kohli, R., R. Krishnamurti. (1989). “Optimal product design using conjoint analysis: Computational complexity and algorithm". Eur. J. Oper. Res. 40(2) 186-195
Lamos H, Galvan S, Gonzalez L, Cruz C (2013). "Algoritmo PSO-Híbrido para solucionar el problema de ruteo de vehículos con entrega y recolección simultáneas". Revista Facultad de Ingeniería. Vol22.

Lamos H, Ariza S, Carvajal E (2014). "Modelo para la solución al problema de diseño de territorios comerciales mediante EPSO". Proyecto de Grado. UIS.

Xiao N(2006) "An evolutionary algorithm for site search problems". Georgr Anal 38(3): 227247

Salazar M, Ríos-Mercado R, Cabrera M, (2011). "New Models for Commercial Territory Design", [en línea]. Springer Science+Business Media.

Wang X., Camm J and D. Curry. (2009) "A Branch-and-Price Approach to the Share-ofChoice Product Line Design Problem". MANAGEMENT SCIENCE. Vol. 55, No. 10, October, pp. 1718-1728.

J.-F. Chen and T.-H. Wu, (2005) "Vehicle routing problem with simultaneous deliveries and pickups," J. Oper. Res. Soc., vol. 57, no. 5, pp. 579-587, Jul..

Tang F. A, Montané and Galvão R, (2006). "A tabu search algorithm for the vehicle routing problem with simultaneous pick-up and delivery service," Comput. Oper. Res., vol. 33, no. 3, pp. 595-619.

Rios-Mercado R.Z, Fernandez EA (2009). “A reactive GRASP for a commercial territory design problem with multiple balancing requirements". Computers \& Operations Research, 36(3), (2009); p. 755-776

Zufryden, F. S. 1982. "Product line optimization by integer programming". Proc. Annual Meeting of ORSA/TIMS, San Diego. 